Check for updates

Cite this: Chem. Commun., 2019, 55, 12960

Received 5th September 2019, Accepted 3rd October 2019

DOI: $10.1039 / \mathrm{c} 9 \mathrm{cc} 06929 a$

rsc.li/chemcomm

\section{Highly chemoselective difluoromethylative homologation of iso(thio)cyanates: expeditious access to unprecedented $\alpha, \alpha$-difluoro(thio)amides $\dagger$}

\author{
Margherita Miele, ${ }^{a}$ Rosarita D'Orsi, ${ }^{b}$ Vellaisamy Sridharan, (D) ${ }^{c}$ Wolfgang Holzer ${ }^{a}$ \\ and Vittorio Pace (D)*a
}

The new motif - $\alpha, \alpha$-difluoromethyl thioamide - has been assembled starting from isothiocyanate (as thioamide precursor) and a formal difluoromethyl-carbanion generated from commercially available $\mathrm{TMSCHF}_{2}$. Upon proper activation of this reagent with potassium tert-amylate, the high-yielding transfer of the difluorinated nucleophile takes place under high chemocontrol. Various sensitive functionalities (e.g. ester, nitrile, nitro, azido groups) can be accommodated across the isothiocyanate core, thus allowing a wide scope. The methodology is highly flexible and adaptable to prepare analogous $\alpha, \alpha$-difluoromethyl oxoamides by conveniently using isocyanates as the electrophilic building-blocks.

Chemical entities featuring a difluoromethyl substituent $\left(\mathrm{CHF}_{2}\right)$ are receiving prominent interest within different audiences because of the fine modulation of physico-chemical parameters achievable by its introduction into organic skeletons. ${ }^{1}$ The relatively acidic proton confers a unique capability to establish $\mathrm{H}$-bond phenomena; ${ }^{2}$ moreover, the $\mathrm{CHF}_{2}$-group acts as a valuable - more lipophilic isostere - of important moieties such as $\mathrm{OH}$ or $\mathrm{SH}$, inter alia. ${ }^{3}$ These properties are regarded as highly valuable during drug optimization processes, in which the continued demand for $\mathrm{CHF}_{2}$-containing scaffolds has boosted the design of novel tactics for their preparation. ${ }^{4}$ In the frame of a medicinal chemistry project, we became interested in preparing $\alpha, \alpha$-difluoromethyl thioamides - completely unprecedented scaffolds - conjugating the versatility of the $\mathrm{CHF}_{2}$ group with unique structural/chemical aspects (stability, crystallizability, good reactivity towards nucleophiles or placeholders for heterocycles) conferred by the formal replacement of oxygen (in oxoamides) with sulfur (Scheme 1). ${ }^{5}$

\footnotetext{
${ }^{a}$ Department of Pharmaceutical Chemistry, University of Vienna, Althanstrasse 14, A-1090, Vienna, Austria. E-mail: vittorio.pace@univie.ac.at; Web: http://drugsynthesis.univie.ac.at/

${ }^{b}$ Department of Sciences, University of Basilicata, Via dell'Ateneo Lucano 10, 85100 Potenza, Italy

${ }^{c}$ Department of Chemistry and Chemical Sciences, Central University of Jammu, Rahya-Suchani (Bagla), 181143, India

$\dagger$ Electronic supplementary information (ESI) available. See DOI: 10.1039/c9cc06929a
}

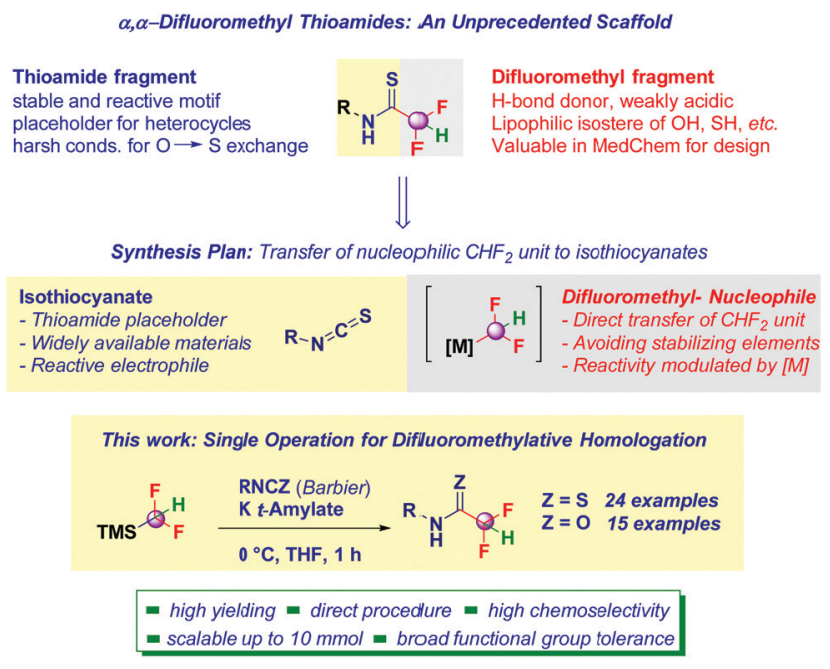

Scheme 1 General context of the presented work.

At the outset of our investigations, we were cognizant of the stability risk of $\alpha$-halogenated thioamides, ${ }^{6}$ thus requiring a straightforward synthetic route - ideally high yielding - to be adopted, in which critical steps such as the formal thionation ( $\mathrm{O} \rightarrow \mathrm{S}$ substitution) could be advantageously skipped. For this purpose, we conceived an approach dealing with the transfer of a putative nucleophilic $\mathrm{CHF}_{2}$-unit to isothiocyanates - as (thio)amides precursors - which proved to be highly effective in addition processes, ${ }^{6,7}$ thus enabling the limitations observed with thionating reagents (e.g. Lawesson) to be circumvented. ${ }^{8}$ Because of the excellent electrophilicity of the heterocumulene carbon a wide array of nucleophilic elements could be installed, thus providing a reliable technique operating under chemocontrol and mild reaction conditions. ${ }^{9}$ However, the wellknown instability of F-containing carbanions ${ }^{10}$ posed important risks on the success of the technique. In fact, to make the strategy productive, two key requirements had to be fulfilled: (1) the conditions to generate the formal $\mathrm{CHF}_{2}$-carbanion must ensure its (limited) chemical integrity and, (2) the per se reactive 
isothiocyanate had to be fully preserved during the carbanion generation event.

The direct use of $\mathrm{MCHF}_{2}$ reagents $(\mathrm{M}=$ metal $)$, limited in terms of versatility and toxicity, stimulated the development of alternative pro-nucleophilic sources for the difluoromethyl anion. ${ }^{10 a}$ In fact, through the positioning of the TMS-group on the putative carbanion, ${ }^{11} \mathrm{Hu}$ and co-workers demonstrated the effectiveness of employing $\mathrm{TMSCHF}_{2}$ in difluoromethylation under nucleophilic regime of carbonyls (ketones and aldehydes) and imines. ${ }^{12}$ Remarkably, it manifests a series of positive features including easy manipulability (bp $65{ }^{\circ} \mathrm{C}$ ) and, the protecting TMS group is advantageously cleaved during the activation event imposed by the presence of the strong $\mathrm{Si}-\mathrm{CF}_{2} \mathrm{H}$ bond. This is a particularly attractive characteristic compared to the use of different protecting elements, mainly strong electronwithdrawing groups, ${ }^{13}$ which would have required two additional undesirable operations: the installation and the removal. An analogous approach has been documented by Dilman who demonstrated the effectiveness of difluorinated phosphorus ylides as nucleophilic reagents. ${ }^{14}$ Herein, we present a straightforward preparation of $\alpha, \alpha$-difluoromethyl thioamides consisting of the nucleophilic $\mathrm{CHF}_{2}$-transfer agent generation/chemoselective addition to isothiocyanates. We anticipate that the strategy is modular and adaptable to the synthesis of analogous $\alpha, \alpha$-difluoromethyl oxoamide derivatives by simply switching to isocyanates as the starting materials. ${ }^{7 a, 15}$

With the aim of verifying the initial hypothesis and evaluating the chemoselectivity of the transformation, the challenging estersubstituted isothiocyanate $\mathbf{1}$ - potentially susceptible of nucleophilic addition at the carbonyl - was selected as the model substrate (Table 1). The activation of $\mathrm{TMSCHF}_{2}$ with CsF was inefficient regardless of the solvent used, thus indicating a distinct behaviour - governed by the nature of the electrophile compared to Hu's work on carbonyls (entries 1 and 2). ${ }^{12}$ Potassium tert-butoxide activated the pro-nucleophile enabling the desired transformation, though significant attack of the
$t$-BuO anion on the isothiocyanate was observed (entry 3). Unfortunately, cooling the reaction at $-78{ }^{\circ} \mathrm{C}$ did not suppress the side product formation (entry 4); interestingly, by switching the solvent to toluene or CPME an increase of the undesired thiocarbamate was noticed (entries 5 and 6). These initial indications suggested us to activate the donor with a more sterically hindered alkoxide, the commercially available tertamylate being a valid alternative. ${ }^{16}$ Pleasingly, the desired difluoromethylthioamide 2 was obtained in high yield as the major product together with a minimal amount of thiocarbamate $\mathbf{2 b}$ (entry 7). Additional considerations merit mention: (1) by decreasing the nucleophile loading to 1.2 equiv. complete suppression of the undesired product was achieved, thus allowing 2 to be prepared with high chemocontrol (entry 8); (2) the putative carbanion needs to be generated under Barbier-type conditions, since late addition (even after $1 \mathrm{~min}$ at $-78{ }^{\circ} \mathrm{C}$ ) of the electrophile results in destruction of the species (entries 9 and 10); (3) the reaction is quite fast reaching completion within $1 \mathrm{~h}$ at $0{ }^{\circ} \mathrm{C}$. Moreover, the use of a small excess of $\mathrm{TMSCHF}_{2}$ compared to $t$-AmOK ( 0.3 equiv.) guarantees the nucleophile generation event to proceed quantitatively (entry 8 vs. 11).

With the optimized conditions in hand (entry 8 - Table 1), we then studied the scope of the reaction (Scheme 2). Not only a sensitive ester (2) could be placed on the reactive aryl isothiocyanate core, but also additional susceptible electrophilic functionalities such as nitrile (3-4) and nitro (5) which remained completely untouched during the nucleophile formation/ homologation sequence. Moreover, nitrogen-containing groups did not interfere with the transformation, as deduced in the case of azide (6) and diazo (7). Different halogens - bromine (8-9 - eventually performing the reaction in $10 \mathrm{mmol}$ scale), fluorine (10), chlorine (11-12) - decorating the aryl nucleus at diverse positions are tolerated, thus allowing their reactivity in late transformations (vide infra) to be exploited. Moving to substituents of opposite electronic behaviour (electron donating groups) alkoxy (12-13) or alkylmercapto (14) - resulted in clean reactions of

Table 1 Reaction optimization

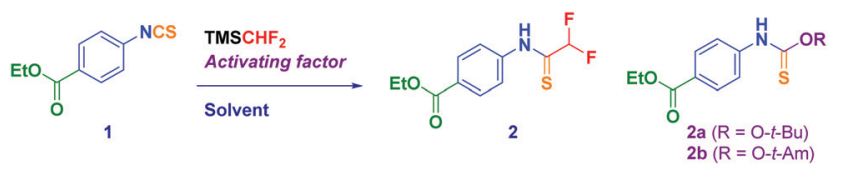

\begin{tabular}{|c|c|c|c|c|c|c|}
\hline Entry & Activator (equiv.) & $\mathrm{TMSCHF}_{2}$ (equiv.) & Solvent & Temperature $\left[{ }^{\circ} \mathrm{C}\right]$ & Ratio $\mathbf{2} / \mathbf{2} \mathbf{a b} \mathbf{b}^{a}$ & Yield of $2^{b}(\%)$ \\
\hline 1 & $\operatorname{CsF}(2.0)$ & 1.8 & DMF & $\mathrm{rt}$ & - & - \\
\hline 2 & CsF (2.0) & 1.8 & THF & $\mathrm{rt}$ & - & - \\
\hline 3 & $t$-BuOK (1.9) & 2.0 & THF & 0 & $70: 30$ & $61^{c}$ \\
\hline 5 & $t$-BuOK (1.9) & 2.0 & Toluene & 0 & $58: 42$ & $47^{e}$ \\
\hline 6 & $t$-BuOK (1.9) & 2.0 & CPME & 0 & $55: 45$ & $41^{f}$ \\
\hline 7 & $t$-AmOK (1.9) & 2.0 & THF & 0 & $88: 12$ & $72^{g}$ \\
\hline 11 & $t$-AmOK (1.2) & 1.2 & THF & 0 & $93: 7^{i}$ & 67 \\
\hline
\end{tabular}

${ }^{a}$ The ratio has been calculated by ${ }^{1} \mathrm{H}$-NMR analysis using 1,3,5-trimethylbenzene as an internal standard. ${ }^{b}$ Isolated yield. ${ }^{c} 2 \mathbf{a}(20 \%) .{ }^{d} 2 \mathbf{2 a}(14 \%) .{ }^{e} 2 \mathrm{a}(31 \%)$.

${ }^{f} \mathbf{2 a}(32 \%){ }^{g} \mathbf{2 b}(9 \%) .{ }^{h}$ Non-Barbier conditions were used (i.e. the nucleophile was generated 1 min prior to the addition of $\left.\mathbf{1}\right){ }^{i} \mathbf{2 b}(4 \%)$. 


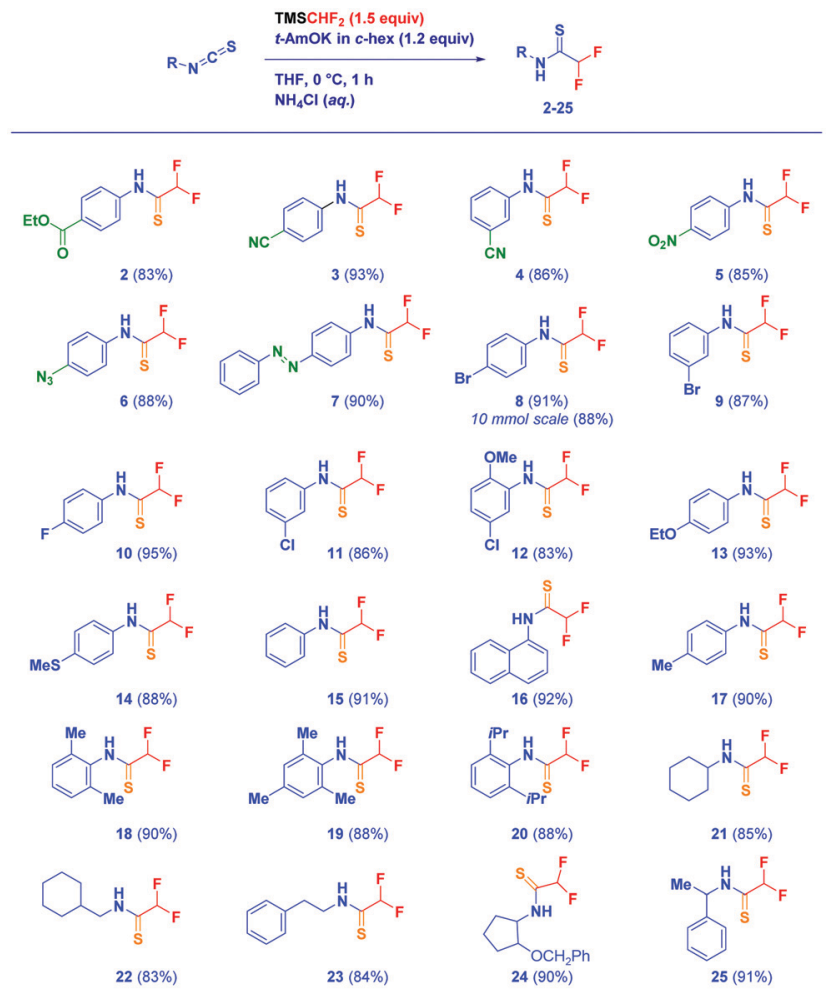

Scheme 2 Scope of the nucleophilic difluoromethylation of isothiocyanates.

comparable efficiency. Moreover, simple aromatic groups - phenyl, $p$-tolyl $(\mathbf{1 5}, \mathbf{1 7})$ or 1-naphthyl $(\mathbf{1 6})$ - provide the expected difluoromethyl thioamides in excellent yields. An intriguing and significantly valuable opportunity of the methodology is the one-step construction of highly sterically demanding scaffolds: accordingly, the introduction of methyl groups at the critical 2 and 6 positions of the phenyl moiety documents the validity of the tactic giving analogues 18 and 19. This aspect is further showcased in the case of the more crowded 2,6-di-i-propylphenyl analogue 20. The nature of the isothiocyanate does not influence the effectiveness of the technique: also aliphatic materials undergo the difluoromethylation giving the corresponding thioamides in high yields (21-25), thus confirming the positive trend manifested by aromatic counterparts.

The success of the procedure motivated us to evaluate the reactivity of oxo-analogues (isocyanates) en route to valuable $\alpha, \alpha$-difluoromethyl amides, synthetically and structurally relevant scaffolds,${ }^{17}$ previously prepared via classical amide-linkage forging chemistry. ${ }^{18}$ The transformation is perfectly flexible affording the targeted motifs in high yield under full chemocontrol (Scheme 3). A series of functionalized aryl isocyanates are amenable for the direct difluoromethylation, including cyano (26), halogen-containing (27-29), alkoxy (30-33), and alkylthio (34). Again, scaling up to $10 \mathrm{mmol}$ is compatible with the protocol (28). Clear confirmation of the suitability of the methodology for preparing challenging sterically demanding substrates arises from the outcome noticed with examples 36 and 37 (aromatic). Additionally, aliphatic congeners afford difluoromethyl amides with similar efficiency, it being worth
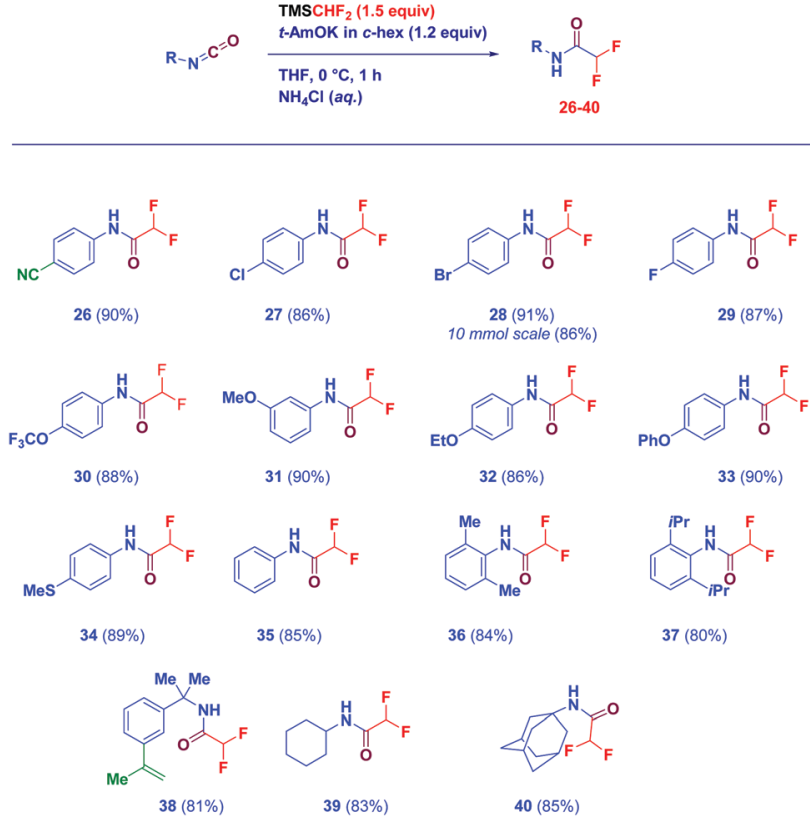

Scheme 3 Synthesis of $\alpha, \alpha$-difluoromethyl amides starting from isocyanates.

noting the congested vinyl-substituted (38) and the adamantyl derivative $\mathbf{4 0}$.

With the aim to explore the potential behaviour of the $\alpha, \alpha$-difluoromethyl thioamide motif, we realized a series of manipulations on the synthesized scaffolds (Scheme 4). Notably, the new motif withstands the Feringa Pd-catalyzed cross-coupling ${ }^{19}$ of $\mathrm{PhLi}$, thus allowing the phenylation on the bromine-containing nucleus 8 to be selectively conducted giving the bis-phenylated structure $\mathbf{4 1}$ (path a). Under alkylative conditions (MeI, KOH), the difluoromethyl thioamide group of $\mathbf{2 0}$ is conveniently converted into the thioimidate 42 (path b). Because of our interest towards $\mathrm{C}_{1}-\mathrm{Sn}$

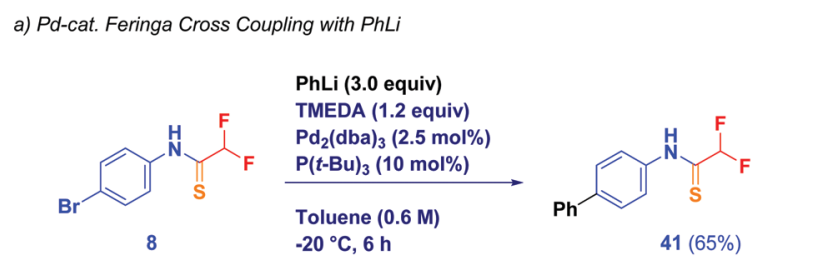

b) Thioamide - thioimidate conversion

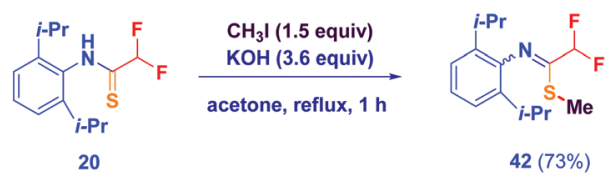

c) Stille-coupling with Me-stannatrane

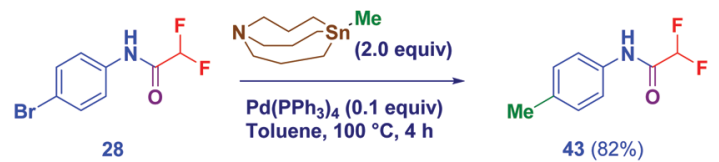

Scheme 4 Synthetic versatility of $\alpha, \alpha$-difluoro(thio)amides. 
reagents, ${ }^{20}$ we were pleased to use a stannatrane ${ }^{21}$ for the transfer of the methyl unit to oxoamide $\mathbf{2 8}$ under Stille-coupling conditions, thus evidencing the stability of the difluoromethyl amide $\mathbf{4 3}$ (path c).

In summary, we have developed a straightforward synthesis of previously undisclosed $\alpha, \alpha$-difluoromethyl thioamides via a tactic based on the nucleophilic transfer of a difluoromethyl carbanion equivalent to isothiocyanates. The treatment of the commercially available and experimentally convenient reagent $\mathrm{TMSCHF}_{2}$ with potassium tert-amylate allows the efficient formation of the difluorinated nucleophile. Full chemocontrol is uniformly manifested as indicated in the cases of challenging isothiocyanates embodying chemical moieties susceptible of nucleophilic attack. This high yielding methodology fully preserves its effectiveness when applied to the synthesis of $\alpha, \alpha$-difluoromethyl oxoamide using isocyanates as amide precursors.

We thank the University of Vienna and the OEAD for generous support. Dr R. Bartolotta is acknowledged for initial contributions and D. Dobusch for HRMS data. R. D'O. thanks the University of Basilicata for a visiting doctoral fellowship. This paper is dedicated to Professor Marko Mihovilovic in the occasion of his 50th birthday.

\section{Conflicts of interest}

There are no conflicts to declare.

\section{Notes and references}

1 For excellent reviews, see: (a) J. Wang, M. Sánchez-Roselló, J. L. Aceña, C. del Pozo, A. E. Sorochinsky, S. Fustero, V. A. Soloshonok and H. Liu, Chem. Rev., 2014, 114, 2432; (b) R. Ragni, A. Punzi, F. Babudri and G. M. Farinola, Eur. J. Org. Chem., 2018, 3500.

2 C. D. Sessler, M. Rahm, S. Becker, J. M. Goldberg, F. Wang and S. J. Lippard, J. Am. Chem. Soc., 2017, 139, 9325.

3 (a) Y. Zafrani, G. Sod-Moriah, D. Yeffet, A. Berliner, D. Amir, D. Marciano, S. Elias, S. Katalan, N. Ashkenazi, M. Madmon, E. Gershonov and S. Saphier, J. Med. Chem., 2019, 62, 5628; (b) Y. Zafrani, D. Yeffet, G. Sod-Moriah, A. Berliner, D. Amir, D. Marciano, E. Gershonov and S. Saphier, J. Med. Chem., 2017, 60, 797.

4 For excellent reviews, see: (a) D. E. Yerien, S. Barata-Vallejo and A. Postigo, Chem. - Eur. J., 2017, 23, 14676; (b) M.-C. Belhomme, T. Besset, T. Poisson and X. Pannecoucke, Chem. - Eur. J., 2015, 21, 12836; (c) J. Rong, C. Ni and J. Hu, Asian J. Org. Chem., 2017, 6, 139. For outstanding recent contributions in the area, see: (d) C. F. Meyer, S. M. Hell, A. Misale, A. A. Trabanco and V. Gouverneur, Angew. Chem., Int. Ed., 2019, 58, 8829; (e) Q. Xie, Z. Zhu, L. Li, C. Ni and J. Hu, Angew. Chem., Int. Ed., 2019, 58, 6405; $(f)$ F. Scheidt, M. Schäfer, J. C. Sarie, C. G. Daniliuc, J. J. Molloy and R. Gilmour, Angew. Chem., Int. Ed., 2018, 57, 16431; $(g)$ F. Pan, G. B. Boursalian and T. Ritter, Angew. Chem., Int. Ed., 2018, 57, 16871; (h) C. Xu, W.-H. Guo, X. He, Y.-L. Guo, X.-Y. Zhang and X. Zhang, Nat. Commun., 2018, 9, 1170.

5 (a) T. S. Jagodziński, Chem. Rev., 2003, 103, 197; (b) T. Murai, Top. Curr. Chem., 2005, 251, 247; (c) Chemistry of Thioamides, ed. T. Murai, Springer Nature, Singapore, 2019; (d) K.-J. Xiao, J.-M. Luo, K.-Y. Ye, Y. Wang and P.-Q. Huang, Angew. Chem., 2010, 122, 3101; (e) K.-J. Xiao, A.-E. Wang and P.-Q. Huang, Angew. Chem., 2012, 124, 8439; $(f)$ V. Pace,
W. Holzer and B. Olofsson, Adv. Synth. Catal., 2014, 356, 3697; (g) V. Pace and W. Holzer, Aust. J. Chem., 2013, 66, 507.

6 (a) B. Yde, N. M. Yousif, U. Pedersen, I. Thomsen and S. O. Lawesson, Tetrahedron, 1984, 40, 2047; (b) L. Castoldi, S. Monticelli, R. Senatore, L. Ielo and V. Pace, Chem. Commun., 2018, 54, 6692.

7 (a) V. Pace, S. Monticelli, K. de la Vega-Hernández and L. Castoldi, Org. Biomol. Chem., 2016, 14, 7848; (b) V. Pace, L. Castoldi, S. Monticelli, M. Rui and S. Collina, Synlett, 2017, 879. For additional studies from our group on the addition of $\alpha$-substituted organometallic reagents, see for example: $(c)$ R. Senatore, L. Castoldi, L. Ielo, W. Holzer and V. Pace, Org. Lett., 2018, 20, 2685; (d) R. Senatore, L. Ielo, E. Urban, W. Holzer and V. Pace, Eur. J. Org. Chem., 2018, 2466; (e) L. Castoldi, L. Ielo, P. Hoyos, M. J. Hernáiz, L. De Luca, A. R. Alcántara, W. Holzer and V. Pace, Tetrahedron, 2018, 74, 2211; $(f)$ R. Senatore, L. Ielo, S. Monticelli, L. Castoldi and V. Pace, Synthesis, 2019, 2792.

8 T. Ozturk, E. Ertas and O. Mert, Chem. Rev., 2007, 107, 5210.

9 (a) V. Pace, L. Castoldi, S. Monticelli, S. Safranek, A. Roller, T. Langer and W. Holzer, Chem. - Eur. J., 2015, 21, 18966; (b) K. de la VegaHernández, R. Senatore, M. Miele, E. Urban, W. Holzer and V. Pace, Org. Biomol. Chem., 2019, 17, 1970.

10 (a) J. Hu, W. Zhang and F. Wang, Chem. Commun., 2009, 7465; (b) A. D. Dilman and V. V. Levin, Acc. Chem. Res., 2018, 51, 1272. For uses of fluorinated carbenoids $\left(\mathrm{LiCH}_{2} \mathrm{~F}\right.$ and LiCHIF) from our group, see: (c) S. Monticelli, M. Colella, V. Pillari, A. Tota, T. Langer, W. Holzer, L. Degennaro, R. Luisi and V. Pace, Org. Lett., 2019, 21, 584; (d) G. Parisi, M. Colella, S. Monticelli, G. Romanazzi, W. Holzer, T. Langer, L. Degennaro, V. Pace and R. Luisi, J. Am. Chem. Soc., 2017, 139, 13648; (e) L. Ielo, S. Touqeer, A. Roller, T. Langer, W. Holzer and V. Pace, Angew. Chem., Int. Ed., 2019, $58,2479$.

11 T. Hagiwara and T. Fuchikami, Synlett, 1995, 717.

12 Y. Zhao, W. Huang, J. Zheng and J. Hu, Org. Lett., 2011, 13, 5342.

13 G. K. S. Prakash and J. Hu, Acc. Chem. Res., 2007, 40, 921.

14 (a) S. S. Ashirbaev, V. V. Levin, M. I. Struchkova and A. D. Dilman, J. Org. Chem., 2018, 83, 478; (b) A. L. Trifonov, V. V. Levin, M. I. Struchkova and A. D. Dilman, Org. Lett., 2017, 19, 5304.

15 For an excellent review, see: (a) E. Serrano and R. Martin, Eur. J. Org. Chem., 2018, 3051. For recent outstanding contributions in the area, see: (b) G. Schäfer, C. Matthey and J. W. Bode, Angew. Chem., Int. Ed., 2012, 51, 9173; (c) X. Wang, M. Nakajima, E. Serrano and R. Martin, J. Am. Chem. Soc., 2016, 138, 15531; (d) S. Zheng, D. N. Primer and G. A. Molander, ACS Catal., 2017, 7, 7957; (e) J. D. Williams, W. J. Kerr, S. G. Leach and D. M. Lindsay, Angew. Chem., Int. Ed., 2018, 57, 12126; $(f)$ D. E. Polat, D. D. Brzezinski and A. M. Beauchemin, Org. Lett., 2019, 21, 4849; (g) J. S. Derasp and A. M. Beauchemin, ACS Catal., 2019, 8104; (h) V. Pace, L. Castoldi and W. Holzer, Chem. Commun., 2013, 49, 8383; $(i)$ V. Pace, K. de la Vega-Hernández, E. Urban and T. Langer, Org. Lett., 2016, 18, 2750.

16 (a) D. D. Caspi and S. M. Richter, J. Org. Chem., 2019, 84, 8256. Recently, we observed an analogous beneficial effect of $\mathrm{K}$ t-amylate in the synthesis of difluoromethyl ketones, see: $(b)$ M. Miele, A. Citarella, N. Micale, W. Holzer and V. Pace, Org. Lett., 2019, DOI: 10.1021/acs.orglett.9b03024.

17 (a) P. J. Czerwiński and B. Furman, Chem. Commun., 2019, 55, 9436; (b) C. R. Jones, P. K. Baruah, A. L. Thompson, S. Scheiner and M. D. Smith, J. Am. Chem. Soc., 2012, 134, 12064.

18 (a) S. S. Biechler and R. W. Taft, J. Am. Chem. Soc., 1957, 79, 4927. For the use of amides in nucleophilic additions, see: $(b)$ M. Nakajima, Y. Oda, T. Wada, R. Minamikawa, K. Shirokane, T. Sato and N. Chida, Chem. - Eur. J., 2014, 20, 17565.

19 (a) M. Giannerini, M. Fañanás-Mastral and B. L. Feringa, Nat. Chem., 2013, 5, 667. For a highlight, see: (b) V. Pace and R. Luisi, ChemCatChem, 2014, 6, 1516.

20 S. Touqeer, L. Castoldi, T. Langer, W. Holzer and V. Pace, Chem. Commun., 2018, 54, 10112.

21 (a) E. Vedejs, A. R. Haight and W. O. Moss, J. Am. Chem. Soc., 1992, 114, 6556; (b) L. Li, C.-Y. Wang, R. Huang and M. R. Biscoe, Nat. Chem., 2013, 5, 607; (c) C.-Y. Wang, G. Ralph, J. Derosa and M. R. Biscoe, Angew. Chem., Int. Ed., 2017, 56, 856. 